\title{
Comparative Evaluation of Pregabalin and Clonidine as Preemptive Analgesics for the Attenuation of Postoperative Pain Following Thoracolumbar Spine Surgery
}

\author{
Renu Bala ${ }^{1}$, Jasbir Kaur ${ }^{2}$, Jyoti Sharma ${ }^{1}$, Raj Singh $^{3}$ \\ ${ }^{1}$ Department of Anaesthesia and Critical Care, Pt. Bhagwat Dayal Sharma Post Graduate Institute of Medical Sciences, Rohtak, India \\ ${ }^{2}$ Department of Anaesthesia, Government Medical College, Chandigarh, India \\ ${ }^{3}$ Department of Orthopaedics, Pt. Bhagwat Dayal Sharma Post Graduate Institute of Medical Sciences, Rohtak, India
}

\begin{abstract}
Study Design: Prospective, randomized, double blind, placebo-controlled study.
Purpose: To compare clonidine and pregabalin with placebo for the attenuation of postoperative pain after thoracolumbar spinal surgery and instrumentation

Overview of Literature: Spine surgery is associated with moderate to severe postoperative pain that needs to be controlled to improve patient's outcome. Alpha 2 agonists (e.g., clonidine) and gabapentenoids (e.g., pregabalin) are successfully used as part of a multimodal analgesic regimen.

Methods: Total 75 patients were enrolled and randomly allocated into three groups. Group P received pregabalin $(150 \mathrm{mg})$, group C received clonidine $(150 \mathrm{mcg}$ ), and group $\mathrm{N}$ received placebo 90 minutes preoperatively. A standard anesthesia protocol comprising fentanyl, thiopentone, vecuronium, nitrous oxide, and oxygen in isoflurane was used for all patients. Postoperative recovery profile, pain, time for first analgesic, 24-hour analgesic requirement, sedation, and hemodynamic parameters were noted.

Results: Recovery profile was similar in all three groups; however, the patients in group $\mathrm{P}$ and $\mathrm{C}$ were more sedated $(p<0.05)$. Group $\mathrm{N}$ patients had a higher Visual Analog Scale (VAS) score $(p<0.05)$ and the time for first analgesic was also lower $(p=0.02)$. Postoperative (24-hour) analgesic requirement was maximum in group $N$, followed by that in group $C$ and group $P$. The VAS score was highest in the control group; however, after 12 hours, it was similar in all groups.

Conclusions: Postoperative pain and analgesic requirement is significantly attenuated by preoperative administration of a single dose of clonidine (150 mcg) or pregabalin (150 mg); pregabalin was more effective. Thus, their use offers a reasonable strategy for pain management in patients undergoing spine surgery.
\end{abstract}

Keywords: Clonidine; Pregabalin; Pain; Thoracolumbar spine; Thoracolumbar spine

\section{Introduction}

Control of postoperative pain is a prime concern in pa- tients undergoing spine surgery because it increases patient comfort, facilitates early mobilization, and improves functional recovery $[1,2]$. Although opioids are the cor-

Received Jan 27, 2019; Revised Mar 17, 2019; Accepted Mar 21, 2019

Corresponding author: Jyoti Sharma

Department of Anaesthesia and Critical Care, Pandit Bhagwat Dayal Sharma Post Graduate Institute Of Medical Sciences, Rohtak, India

Tel: +91-9968583915, E-mail: doctorjyotisharma@yahoo.in 
ner-stone in pain management, they are often associated with myriad complications [3]. A multimodal analgesic regimen wherein 2 or more analgesics of different groups are administered is increasingly being adopted recently [4]. Further, preemptive analgesia treatment wherein antinociceptive therapy is given before surgical stimulus is very effective in these painful surgeries [5].

Clonidine is an alpha 2 agonist that possesses multiple actions, such as sedation, anxiolysis, and analgesia. Its favorable functional profile has encouraged its perioperative use through various routes [6,7]. Pregabalin is a newer gabapentenoid that was originally introduced as an antiepileptic but showed good pain relief against neuropathic pain. In spine surgery, gabapentenoids are successfully used to decrease postoperative pain $[8,9]$. Therefore, we designed the present study to evaluate and compare clonidine and pregabalin as preemptive analgesics in patients undergoing thoracolumbar spine surgery. Our study was based on the null hypothesis that both the drugs will attenuate postoperative pain owing to their analgesic effects.

\section{Materials and Methods}

\section{Patients}

The present prospective, randomized, double blind, placebo control study was conducted in the Department of Anaesthesiology and Critical Care of Pandit Bhagwat Dayal Sharma Post Graduate Institute of Medical Sciences, Rohtak after obtaining approval from the institutional ethics committee of Pandit Bhagwat Dayal Sharma Post Graduate Institute of Medical Sciences, Rohtak (Endst No. Surg. I/16/537-74 dtd 17/2/2016). Seventy-five adult patients (age group, 18-65 years) of either sex, with an American Society of Anesthesiologists (ASA) physical status 1 and 2 and undergoing elective thoracolumbar spinal instrumentation for isolated spinal trauma requiring laminectomy (minimum one level and maximum three levels) were enrolled in the study after they provided informed written consent. Patients who were already on the study drug; had a history of allergy to the study drug; had epilepsy, liver, or kidney diseases; were pregnant or lactating; had a psychiatric illness, drug or alcohol abuse; were on beta blockers; and those who receiving narcotics within 24 hours of the surgery were excluded. Patients with other injuries (chest, abdominal, head, long bones, etc.) were also excluded.
During pre-anesthetic rounds, the detailed clinical history was taken, clinical examination was performed, and anthropometric parameters were evaluated. Routine investigations and special investigations were conducted as per requirement. The purpose and protocol of the study and scoring of pain as per the Visual Analog Scale (VAS) was explained to the patients. Adequate fasting of 6 hours before the surgery was advised, and premedication was given in the form of a 150-mg tablet of ranitidine orally the night before and in the morning 2 hours before the surgery.

Patients were randomly allocated to either of the following three groups using a computer-generated sequence of random numbers: group $\mathrm{P}(\mathrm{n}=25)$ received pregabalin capsule (150 mg, PREGABID-150; Intas Pharmaceuticals Ltd., Dehradun, India), group C $(n=25)$ received clonidine tablet (150 mcg, ARKAMIN-100; Unichem, Mumbai, India), and group $\mathrm{N}(\mathrm{n}=25)$ received placebo (calcium tablet) 90 minutes before the surgery with a sip of water.

On arrival in the operating room, an intravenous line was secured. Routine monitoring, including heart rate (beats per minute), electrocardiogram, non-invasive blood pressure, and pulse oximetry $\left(\mathrm{SpO}_{2}\right)$ was performed using Philips Intellivue MP50 monitor. General anesthesia was induced using fentanyl $2 \mathrm{mcg} \mathrm{kg}^{-1}$, sleep dose of thiopentone (incremental dose was administered till loss of eyelash reflex) was given, and orotracheal intubation was facilitated with vecuronium $0.10 \mathrm{mg} \mathrm{kg}^{-1}$. Intraoperatively, end tidal carbon dioxide, temperature, bispectral index (BIS), and inspired/expired concentration of isoflurane were also monitored.

Before the surgery, the incision site was infiltrated by the surgeon using lignocaine $1 \%$ with adrenaline $1: 2,00,000$. In all the patients, surgery was performed via the open and posterior approach in the prone position. Laminectomy of required levels was performed to perform cord decompression followed by fixation of the spine using pedicle and screw. Anesthesia was maintained with oxygen (35\%) and nitrous oxide $(65 \%)$ in isoflurane (minimum alveolar concentration titrated to keep BIS 40 to 60 ) and intermittent vecuronium $\left(0.02 \mathrm{mg} \mathrm{kg}^{-1}\right)$, whenever indicated. Fluid management and blood transfusion was done as per standard protocol.

Following surgery completion, isoflurane was discontinued after skin suturing, and nitrous oxide was stopped after the patient was made to lie in the supine position. Neuromuscular blockade was reversed with neostigmine $50 \mathrm{mcg} \mathrm{kg}^{-1}$ and glycopyrrolate $10 \mathrm{mcg} \mathrm{kg}^{-1}$, and extuba- 
tion was done when adequate spontaneous ventilation was established. Emergence time (time from switching off nitrous oxide until the opening of eyes), and extubation time (time from switching off nitrous oxide until tracheal extubation) was noted. Patients sedation level (as per Ramsay Sedation Scale), pain at rest and during movement (while coughing) was assessed using the VAS score. Postoperative nausea and vomiting, shivering, or any other complaints, such as dry mouth, visual disturbances, and headache were noted and managed appropriately.

In the recovery room, the patients' recovery was graded as per the Modified Aldrete Score (MAS). A maximum score of 9 was considered because many of our patients had paraplegia, and movement of the lower limbs could not be demonstrated.

Time of giving the first rescue analgesic, defined as the period from surgery to when the first dose of analgesia was administered at patient's request (VAS $>3$ ), was recorded. On pain, fentanyl $1 \mathrm{mcg} \mathrm{kg}^{-1}$ was administered intravenously followed by $0.5 \mathrm{mcg} \mathrm{kg}^{-1}$ in case of VAS $>3$ with a maximum dose of $2 \mathrm{mcg} \mathrm{kg}^{-1}$ in 1 hour. Ketorolac injection $(30 \mathrm{mg}$ ) was given as rescue analgesic and repeated every 8 hours, whenever required. The patients' vital parameters, sedation level, and pain score, both at rest (VAS static) and during movement (VAS dynamic) were recorded at $0,1,2,4,6,12$, and 24 hours. The total dose of analgesics, fentanyl and ketorolac, antiemetic administered in 24 hours was also recorded.

\section{Statistical analyses}

A sample size of 75 patients ( 25 patients in each group) was used to detect a significant difference of $20 \%$ in the fentanyl consumption with a power of $85 \%$ and a significance level of $5 \%$.

The data obtained were compiled and analyzed using IBM SPSS ver. 20.0 (IBM Corp., Armonk, NY, USA). Quantitative data were analyzed using one-way analysis of variance (one-way analysis of variance). Qualitative data were analyzed using chi-square test for the ASA grading, sex, VAS score, and time for first rescue analgesic and Fisher exact test for adverse events. Post-hoc analyses using Bonferroni test was used for multiple comparisons.

Normally distributed data are presented as mean \pm standard deviation values, and categorical data are presented as frequencies and percentages. A $p$-value $<0.05$ was considered significant.

\section{Results}

The demographic profile and American Spinal Injury Association scores of patients in the three groups were

Table 1. Demographic profile and ASIA score of patients

\begin{tabular}{|c|c|c|c|c|}
\hline Variable & Group P ( $n=25)$ & Group C ( $n=25)$ & Group N (n=25) & $p$-value \\
\hline Age (yr) & $31.72 \pm 10.33(20-60)$ & $34.08 \pm 14.34(18-65)$ & $39.76 \pm 12.05(18-60)$ & 0.06 \\
\hline Gender & & & & 0.40 \\
\hline Male & $17(68)$ & $17(68)$ & $13(52)$ & \\
\hline Female & $8(32)$ & $8(32)$ & $12(48)$ & \\
\hline ASA grade & & & & 0.74 \\
\hline Grade 1 & $22(88)$ & $21(84)$ & $20(80)$ & \\
\hline Grade 2 & $3(12)$ & $4(16)$ & $5(20)$ & \\
\hline Height (cm) & $163.16 \pm 4.33(154-172)$ & $163.76 \pm 4.80(152-172)$ & $162.32 \pm 4.90(154-172)$ & 0.55 \\
\hline Weight (kg) & $61.68 \pm 10.09(50-90)$ & $58.96 \pm 8.07(40-70)$ & $62.04 \pm 12.10(45-98)$ & 0.51 \\
\hline Body mass index $\left(\mathrm{kg} / \mathrm{m}^{2}\right)$ & $23.12 \pm 3.45$ & $21.97 \pm 2.76$ & $23.51 \pm 4.20$ & 0.28 \\
\hline ASIA score & & & & 0.993 \\
\hline A & $9(36)$ & $9(36)$ & $8(32)$ & \\
\hline B & $8(32)$ & $7(28)$ & $8(32)$ & \\
\hline C & $4(16)$ & $6(24)$ & $5(20)$ & \\
\hline D & $4(16)$ & $3(12)$ & $4(16)$ & \\
\hline
\end{tabular}

Values are presented as mean \pm standard deviation (range) or number (\%).

ASA, American Society of Anesthesiologists; ASIA, American Spinal Injury Association. 
Table 2. The details regarding surgery are shown

\begin{tabular}{lcccc} 
Variable & Group $P(n=25)$ & Group C $(n=25)$ & Group N (n=25) & $p$-value \\
\hline Duration of surgery $(\mathrm{min})$ & $153.2 \pm 30.0$ & $143.4 \pm 30.4$ & $138.4 \pm 27.9$ & 0.19 \\
\hline No. of laminectomies & & & 13 & 0.961 \\
\hline 1 & 14 & 11 & 10 & 2 \\
\hline 2 & 10 & 1 & $186.3 \pm 75.2$ & 0.52 \\
\hline 3 & 1 & $199.2 \pm 62$ & $1,477.8 \pm 360$ & 0.15 \\
\hline Blood loss $(\mathrm{mL})$ & $213.2 \pm 55.2$ & $1,760.2 \pm 600$ & &
\end{tabular}

Values are presented as mean \pm standard deviation or number, unless otherwise stated.

Table 3. Recovery profile of patients in three groups

\begin{tabular}{lcccc} 
Variable & Group P & Group C & Group N & $p$-value \\
\hline Emergence time $(\mathrm{sec})$ & $358.12 \pm 136.11$ & $342.80 \pm 177.44$ & $289.28 \pm 92.40$ & 0.19 \\
\hline Extubation time $(\mathrm{sec})$ & $335.20 \pm 138.29$ & $291.80 \pm 152.28$ & $344.44 \pm 107.25$ & 0.38 \\
\hline Ramsay Sedation Scale & $2.32 \pm 0.62$ & $2.40 \pm 0.50$ & $1.56 \pm 0.65$ & 0.00 \\
Modified Aldrete Score & $7.50 \pm 0.70$ & $7.64 \pm 0.56$ & $7.60 \pm 0.95$ & 0.13 \\
\hline
\end{tabular}

Values are presented as mean \pm standard deviation.

Table 4. Post-hoc analyses of three groups and $p$-value shown

\begin{tabular}{lccc} 
Variable & Group P vs. group C & Group P vs. group N & Group C vs. group N \\
Emergence time & 1.00 & 0.25 & 0.54 \\
Extubation time & 0.76 & 1.00 & 0.50 \\
\hline Ramsay Sedation Scale & 1.00 & 0.00 & 0.00 \\
Modified Aldrete Score & 0.12 & 0.24 & 1.00 \\
Time for 1st analgesia & 0.397 & 0.023 & 0.001 \\
\hline
\end{tabular}

Table 5. Immediate postoperative pain and analgesic consumption in three groups

\begin{tabular}{|c|c|c|c|c|}
\hline Variable & Group P & Group C & Group N & $p$-value \\
\hline \multicolumn{5}{|l|}{ Visual Analog Scale } \\
\hline Static & $1.46 \pm 0.68$ & $2.67 \pm 1.20$ & $5.24 \pm 1.33$ & 0.00 \\
\hline Dynamic & $2.44 \pm 1.52$ & $3.64 \pm 1.40$ & $5.36 \pm 1.80$ & 0.00 \\
\hline Time for 1 st analgesic (min) & $51.24 \pm 49.96$ & $35.44 \pm 36.66$ & $7.0 \pm 14.77$ & 0.001 \\
\hline
\end{tabular}

Values are presented as mean \pm standard deviation.

comparable, as shown in Table $1(p>0.05)$. The surgical details are shown in Table 2 . The recovery profile was also comparable in the three groups, except the sedation score that was higher in group $\mathrm{P}$ and $\mathrm{C}$ (Table 3); post-hoc analysis of the groups is shown in Table 4. Postoperative pain was greater in the control group and time for first analgesic requirement was maximum in group P (Table
5). More patients in group $\mathrm{N}$ required immediate pain relief, while those in groups $\mathrm{P}$ and $\mathrm{C}$ required delayed pain relief, as shown in Fig. 1. Table 6 shows the postoperative pain scores; this score was higher in group $\mathrm{N}$ until 6 hours after which, it was comparable in all groups. Postoperative analgesic requirement was significantly reduced in group $\mathrm{P}$, as shown in Table 7 . Hemodynamics (heart rate and 


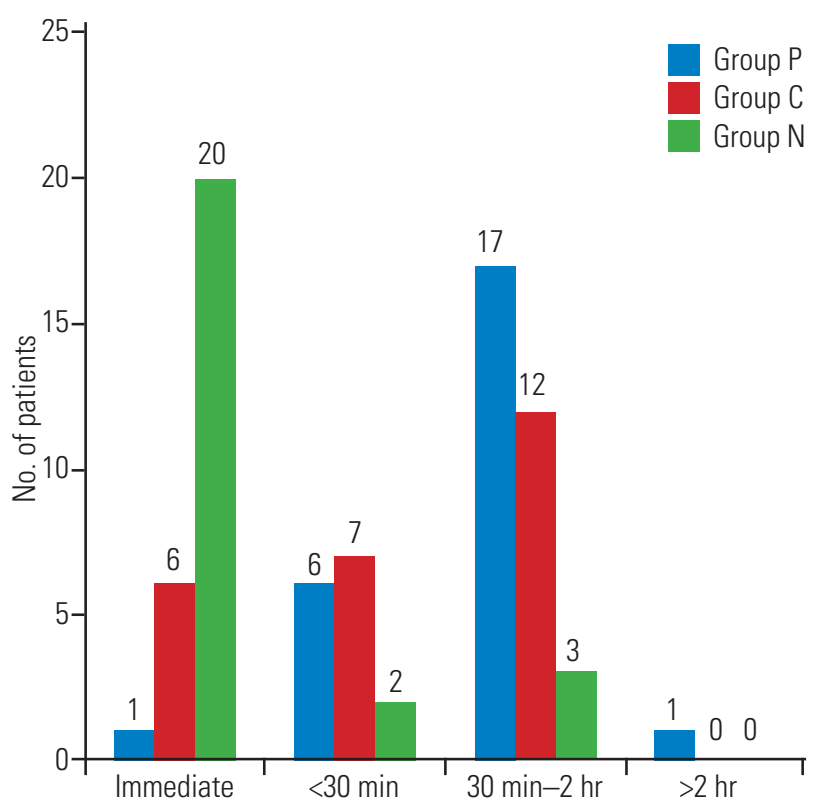

Fig. 1. Distribution of patients according to first analgesic requirement.

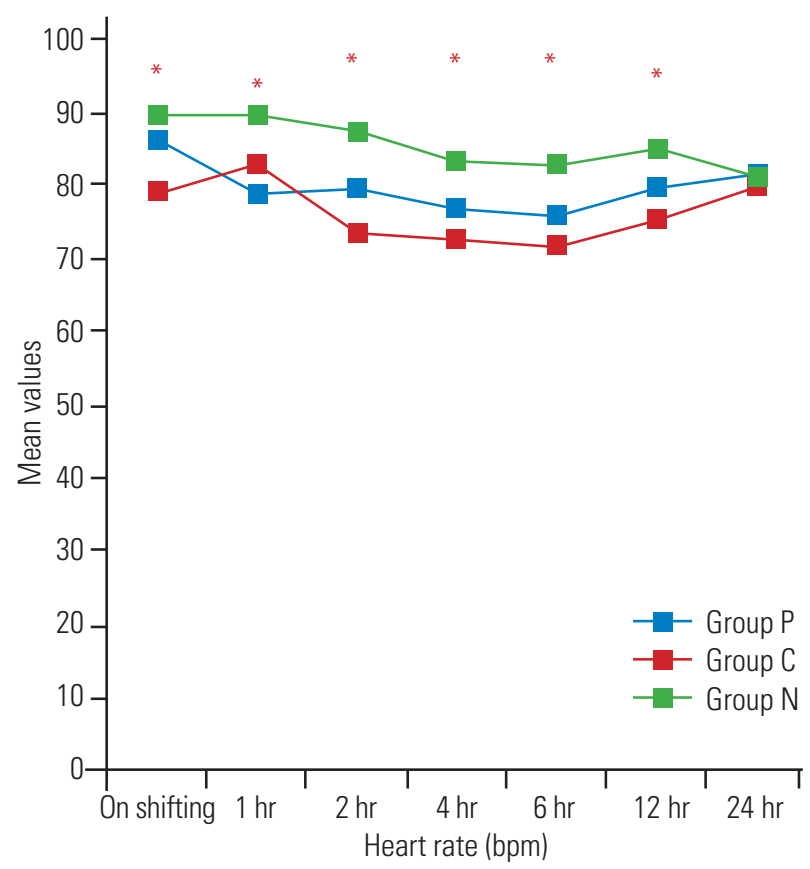

Fig. 2. Postoperative variation in heart rate at various time intervals.

blood pressure) of the patients in the three groups during the 24-hour postoperative period is shown in Figs. 2-4. Although statistically significant at various time intervals, clinically they were in the normal range. We did not observe any adverse event in any patient. The patients in groups $\mathrm{P}$ and $\mathrm{C}$ were more sedated in the first postoperative hour; thereafter, there was no difference in the sedation scores (Table 8).

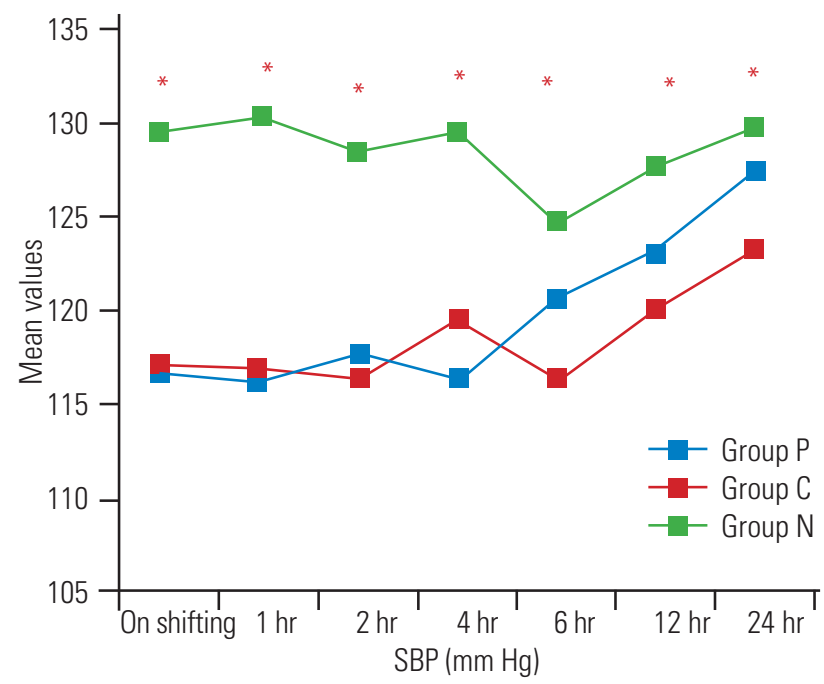

Fig. 3. Postoperative variation in SBP at various time intervals. SBP, systolic blood pressure.

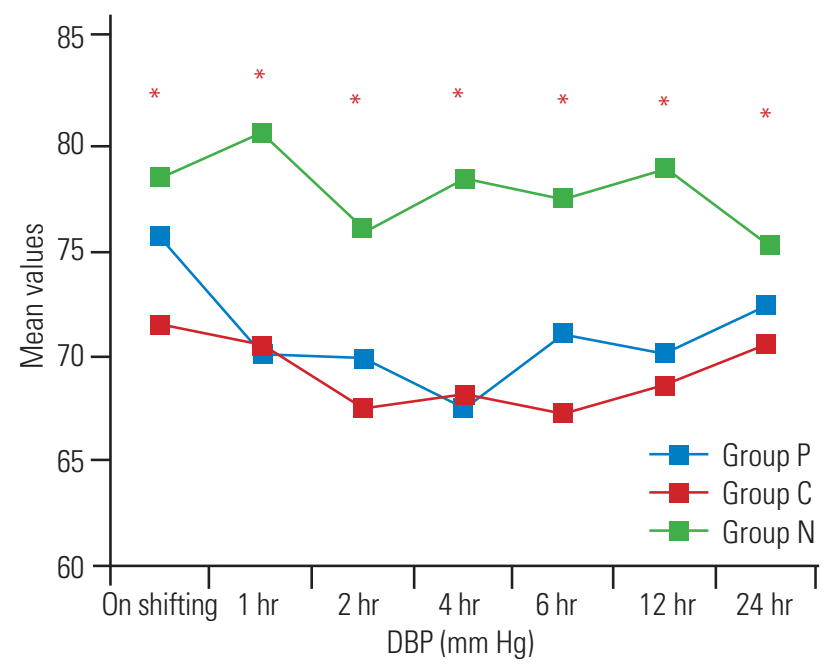

Fig. 4. Postoperative variation in DBP at various time intervals. DBP, diastolic blood pressure.

\section{Discussion}

Pre-emptive analgesia prevents the central processing of afferent input responsible for postoperative pain and subsequently decreases the incidence of hyperalgesia and allodynia after surgery. This technique is highly desirable in surgeries associated with severe postoperative pain $[5,10,11]$. Major spine surgeries, such as spinal instrumentation involve extensive tissue trauma and manipulation of muscles and ligaments intraoperatively. Moreover, operation-induced neuroplastic changes may induce subsequent sensitization leading to postoperative hyperalgesia and allodynia [1,2]. Therefore, we intended 
Table 6. Postoperative VAS in three groups

\begin{tabular}{|c|c|c|c|c|c|}
\hline Variable & Category & Group P & Group C & Group N & $p$-value \\
\hline \multirow[t]{2}{*}{ On shifting } & Vs & $1.89 \pm 0.76$ & $4.40 \pm 0.52$ & $4.80 \pm 0.92$ & 0.00 \\
\hline & $\mathrm{Vd}$ & $2.64 \pm 0.58$ & $4.54 \pm 0.77$ & $5.36 \pm 0.97$ & 0.00 \\
\hline \multirow[t]{2}{*}{$1 \mathrm{hr}$} & Vs & $2.44 \pm 0.49$ & $4.09 \pm 0.45$ & $4.48 \pm 0.47$ & 0.00 \\
\hline & $\mathrm{Vd}$ & $2.78 \pm 0.80$ & $4.53 \pm 0.55$ & $4.80 \pm 1.10$ & 0.00 \\
\hline \multirow[t]{2}{*}{$2 \mathrm{hr}$} & Vs & $2.04 \pm 1.11$ & $3.94 \pm 1.33$ & $4.40 \pm 0.9$ & 0.00 \\
\hline & $\mathrm{Vd}$ & $3.84 \pm 1.25$ & $4.23 \pm 1.16$ & $4.97 \pm 1.03$ & 0.003 \\
\hline \multirow[t]{2}{*}{$4 \mathrm{hr}$} & Vs & $2.56 \pm 0.53$ & $4.16 \pm 0.42$ & $4.66 \pm 0.39$ & 0.00 \\
\hline & $\mathrm{Vd}$ & $3.60 \pm 1.33$ & $4.40 \pm 1.25$ & $4.84 \pm 1.11$ & 0.003 \\
\hline \multirow[t]{2}{*}{$6 \mathrm{hr}$} & Vs & $2.32 \pm 0.68$ & $4.09 \pm 0.78$ & $4.15 \pm 0.50$ & 0.00 \\
\hline & $\mathrm{Vd}$ & $2.72 \pm 0.90$ & $4.43 \pm 0.74$ & $4.88 \pm 0.60$ & 0.00 \\
\hline \multirow[t]{2}{*}{$12 \mathrm{hr}$} & Vs & $1.56 \pm 0.77$ & $1.60 \pm 0.90$ & $1.75 \pm 1.48$ & 0.81 \\
\hline & $\mathrm{Vd}$ & $2.30 \pm 0.97$ & $2.36 \pm 0.68$ & $2.44 \pm 0.90$ & 0.84 \\
\hline \multirow[t]{2}{*}{$24 \mathrm{hr}$} & Vs & $1.50 \pm 0.68$ & $1.30 \pm 0.62$ & $1.34 \pm 0.77$ & 0.80 \\
\hline & $\mathrm{Vd}$ & $2.80 \pm 1.11$ & $2.78 \pm 0.86$ & $2.67 \pm 0.90$ & 0.87 \\
\hline
\end{tabular}

Values are presented as mean \pm standard deviation.

VAS, Visual Analog Scale; Vs, VAS static; Vd, VAS dynamic.

Table 7. Postoperative analgesic requirement

\begin{tabular}{lcccc} 
Variable & Group P & Group C & Group N & $p$-value \\
24-Hour fentanyl requirement (mcg) & $1,890 \pm 660.20$ & $2,080 \pm 810.00$ & $2,720 \pm 980.90$ & 0.002 \\
24-Hour ketorolac (mg) & $41.53 \pm 19.51$ & $60.00 \pm 21.19$ & $63.75 \pm 18.37$ & 0.00 \\
Doses of ketorolac required & 22 & 38 & 50 & 0.001 \\
\hline
\end{tabular}

Values are presented as mean \pm standard deviation or number, unless otherwise stated.

Table 8. Postoperative variation in RSS at various time intervals

\begin{tabular}{lllll} 
RSS & Group P & Group C & Group N & $p$-value \\
\hline On shifting & $2.28 \pm 0.61$ & $2.28 \pm 0.54$ & $1.44 \pm 0.65$ & 0.00 \\
\hline $1 \mathrm{hr}$ & $1.80 \pm 0.40$ & $1.80 \pm 0.40$ & $1.50 \pm 0.50$ & 0.04 \\
\hline $2 \mathrm{hr}$ & $1.76 \pm 0.43$ & $1.56 \pm 0.50$ & $1.68 \pm 0.55$ & 0.37 \\
\hline $\mathrm{hr}$ & $1.64 \pm 0.48$ & $1.40 \pm 0.50$ & $1.40 \pm 0.50$ & 0.15 \\
\hline $6 \mathrm{hr}$ & $1.36 \pm 0.48$ & $1.36 \pm 0.48$ & $1.36 \pm 0.48$ & 1.00 \\
$12 \mathrm{hr}$ & $1.04 \pm 0.20$ & $1.12 \pm 0.33$ & $1.00 \pm 0.0$ & 0.16 \\
\hline $24 \mathrm{hr}$ & $1.00 \pm 0.0$ & $1.04 \pm 0.20$ & $1.04 \pm 0.20$ & 0.09 \\
\hline
\end{tabular}

Values are presented as mean \pm standard deviation.

RSS, Ramsay Sedation Scale score.

to target postoperative pain in this group of patients using gabapentenoid or alpha 2 agonist; both drugs have been used safely as adjuvant analgesics in different surgeries.

Our study agrees with the results of earlier studies that pregabalin and clonidine provide pain relief when used as component of a multimodal analgesic regimen [12-17]. Between these two drugs, pregabalin provided better analgesia compared to clonidine. In the postoperative period, the patients who received the study drug experienced less pain than those who were given placebo until 6 hours. 
However, at 12 hours and beyond, there was no difference in the VAS scores of the three groups.

Pregabalin has earlier been used successfully in spine surgery patients. There were diverse pathologies of patients, ranging from laminectomy and discectomy to spinal instrumentation $[9,18]$. Pregabalin is a structural analog of gamma amino butyric acid introduced in 2004. It binds to alpha 2 delta subunit of voltage-gated calcium channels and prevents the release of pain mediators, such as substance $\mathrm{P}$, noradrenaline, and glutamate. It also exerts antihyperalgesic effects and inhibits central sensitization. Its pharmacokinetic profile is much better than its previous congener, gabapentin. Several types of chronic pain syndromes have been treated with gabapentenoids in addition to their antiepileptic effects [19]. As an adjunct to perioperative analgesics, it has been used in the dose ranging from $50-600 \mathrm{mg}$. A dose $>300 \mathrm{mg}$ achieved greater reduction in the perioperative opioid consumption; nevertheless, the risks of adverse effects, such as dizziness, lightheadedness, and visual disturbances were also more $[8,13]$. Thus, we opted for a $150-\mathrm{mg}$ dose that has safely been used in most studies. Pregabalin has been compared with other agents, such as gabapentin, tramadol, nonsteroidal anti-inflammatory drugs, and celecoxib that are also used as a component of multimodal analgesia $[12,20,21]$. However, we compared it to alpha 2 adrenergic agonist, clonidine. It is a centrally acting antihypertensive that has also been used perioperatively through various routes to reduce postoperative pain. It binds to alpha 2 adrenergic receptors in the spinal cord to stimulate acetylcholine release that acts at both muscarinic and nicotinic subtypes for postoperative pain relief $[6,17]$. Oral clonidine when administered preoperatively to patients undergoing spine surgery reduced postoperative morphine consumption in a previous study [22]. The other alpha 2 agonist, dexmedetomidine, has been successfully used in spine surgery [23]. Pregabalin and clonidine were compared in a previous study comprising surgeries under spinal anesthesia, and pregabalin displayed superior efficacy in alleviating postoperative pain, similar to our results [7].

The time for first analgesia was prolonged in the pregabalin group followed by that in the clonidine and placebo groups, thus indicating a superior analgesic effect of pregabalin. The follow-up period of our study was 24 hours during which, analgesic consumption, hemodynamic parameters, and any adverse effects were assessed. The 24hour fentanyl consumption was minimum in the prega- balin group, followed by that in the clonidine and placebo groups. Our results corroborated with those of previous studies [12-14,20]. Fujita et al. [15] also reported that a single preoperative administration of $150 \mathrm{mg}$ of pregabalin reduced the postoperative pain intensity and morphine consumption in patients who underwent posterior lumbar interbody fusion. However, Choi et al. [24] could not observe any difference in the postoperative remifentanil consumption following pregabalin premedication. The results of a study by Urban et al. [25] are also contradictory to our findings. In a sub-study of the POISE-2 trial (Perioperative Ischemic Evaluation-2 trial), preoperative administration of $200 \mathrm{mg}$ of clonidine in non-cardiac surgery did not attenuate postoperative pain or reduce analgesic consumption, in contrast to our results [26].

Patients' sedation level (as per the Ramsay Sedation Scale) was assessed. The Ramsay Sedation Scale score is one of the most commonly used scales for assessing sedation in routine practice and clinical research. The score can range from 1-6, with 1 indicating that the patient is fully awake with anxiety or restlessness or both and a score of 6 meaning deep sedation with no response to stimulus as in general anesthesia.

In the recovery room, patients' recovery was graded as per the MAS, a scoring system to assess postoperative recovery. Patients were evaluated for various parameters, such as motor activity, respiration, circulation, consciousness, and color; they were also assessed for readiness of discharge from the postoperative care unit. A maximum score of 9 was considered because many patients had paraplegia, and movement of the lower limbs could not be demonstrated.

In the immediate postoperative period, patients who received study drug were more sedated; however, at 2 hours and thereafter, all patients had similar sedation scores. Our results are in agreement with those of Reuben et al. [12]. However, Ozgencil et al. [27] noted more sedation following the use of pregabalin; this may be due to a higher dose $(300 \mathrm{mg})$. Postoperative nausea and vomiting was more common in the placebo group and could be attributed to greater consumption of opioids. Dizziness, a commonly observed complication following pregabalin administration was not present in any patient, potentially because of the low dose of pregabalin used. Our results are in accordance with the findings of recent meta-analyses that report that the preoperative use of pregabalin is efficacious in reducing postoperative pain, opioid consump- 
tion, and nausea following spine surgery $[9,28]$.

Our study had certain limitations. First, the study drugs, pregabalin and clonidine belong to different groups. The most commonly used and the safest dose was selected; however, it is difficult to comment if $150 \mathrm{mg}$ of pregabalin is equivalent to $150 \mathrm{mcg}$ of clonidine in terms of analgesic efficacy. Second, we administered a single dose of the study drugs. Repeated doses have been used in few studies that may provide better postoperative pain relief. Third, our patients were young and had good ASA grades. Safety of these drugs in the older age group and those in worse clinical condition is unclear. Fourth, long-term functional outcomes were not assessed.

\section{Conclusions}

We conclude that the administration of a single dose of pregabalin or clonidine as pre-emptive analgesia is an effective, reasonable, and safe strategy in patients undergoing spinal instrumentation for reducing postoperative pain and analgesic consumption. Further, pregabalin is superior to clonidine. Further trials, comprising more number of patients are warranted.

\section{Conflict of Interest}

No potential conflict of interest relevant to this article was reported.

\section{References}

1. Bajwa SJ, Haldar R. Pain management following spinal surgeries: an appraisal of the available options. J Craniovertebr Junction Spine 2015;6:105-10.

2. Nolte MT, Elboghdady IM, Iyer S. Anesthesia and postoperative pain control following spine surgery. Semin Spine Surg 2018;30:154-9. https://10.1053/ j.semss.2018.07.013.

3. Radhakrishnan M, Bithal PK, Chaturvedi A. Effect of preemptive gabapentin on postoperative pain relief and morphine consumption following lumbar laminectomy and discectomy: a randomized, doubleblinded, placebo-controlled study. J Neurosurg Anesthesiol 2005; $17: 125-8$.

4. Devin CJ, McGirt MJ. Best evidence in multimodal pain management in spine surgery and means of assessing postoperative pain and functional outcomes.
J Clin Neurosci 2015;22:930-8.

5. Sekar C, Rajasekaran S, Kannan R, Reddy S, Shetty TA, Pithwa YK. Preemptive analgesia for postoperative pain relief in lumbosacral spine surgeries: a randomized controlled trial. Spine J 2004;4:261-4.

6. Marinangeli F, Ciccozzi A, Donatelli F, et al. Clonidine for treatment of postoperative pain: a dosefinding study. Eur J Pain 2002;6:35-42.

7. Prasad A, Bhattacharyya S, Biswas A, Saha M, Mondal S, Saha D. A comparative study of pre-operative oral clonidine and pregabalin on post-operative analgesia after spinal anesthesia. Anesth Essays Res 2014;8:41-7.

8. Zhang J, Ho KY, Wang Y. Efficacy of pregabalin in acute postoperative pain: a meta-analysis. Br J Anaesth 2011;106:454-62.

9. Liu B, Liu R, Wang L. A meta-analysis of the preoperative use of gabapentinoids for the treatment of acute postoperative pain following spinal surgery. Medicine (Baltimore) 2017;96:e8031.

10. Kissin I. Preemptive analgesia. Anesthesiology 2000;93:1138-43.

11. Ong CK, Lirk P, Seymour RA, Jenkins BJ. The efficacy of preemptive analgesia for acute postoperative pain management: a meta-analysis. Anesth Analg 2005;100:757-73.

12. Reuben SS, Buvanendran A, Kroin JS, Raghunathan $\mathrm{K}$. The analgesic efficacy of celecoxib, pregabalin, and their combination for spinal fusion surgery. Anesth Analg 2006;103:1271-7.

13. Kim JC, Choi YS, Kim KN, Shim JK, Lee JY, Kwak YL. Effective dose of peri-operative oral pregabalin as an adjunct to multimodal analgesic regimen in lumbar spinal fusion surgery. Spine (Phila Pa 1976) 2011;36:428-33.

14. Gianesello L, Pavoni V, Barboni E, Galeotti I, Nella A. Perioperative pregabalin for postoperative pain control and quality of life after major spinal surgery. J Neurosurg Anesthesiol 2012;24:121-6.

15. Fujita N, Tobe M, Tsukamoto N, Saito S, Obata H. A randomized placebo-controlled study of preoperative pregabalin for postoperative analgesia in patients with spinal surgery. J Clin Anesth 2016;31:149-53.

16. Mohammadi SS, Seyedi M. Comparing oral gabapentin versus clonidine as premedication on early postoperative pain, nausea and vomiting following general anesthesia. Saudi J Anaesth 2009;3:25-8. 
17. Singh S, Arora K. Effect of oral clonidine premedication on perioperative haemodynamic response and postoperative analgesic requirement for patients undergoing laparoscopic cholecystectomy. Indian J Anaesth 2011;55:26-30.

18. Jiang HL, Huang S, Song J, Wang X, Cao ZS. Preoperative use of pregabalin for acute pain in spine surgery: a meta-analysis of randomized controlled trials. Medicine (Baltimore) 2017;96:e6129.

19. Bockbrader HN, Wesche D, Miller R, Chapel S, Janiczek N, Burger P. A comparison of the pharmacokinetics and pharmacodynamics of pregabalin and gabapentin. Clin Pharmacokinet 2010;49:661-9.

20. Khurana G, Jindal P, Sharma JP, Bansal KK. Postoperative pain and long-term functional outcome after administration of gabapentin and pregabalin in patients undergoing spinal surgery. Spine (Phila Pa 1976) 2014;39:E363-8.

21. Kumar KP, Kulkarni DK, Gurajala I, Gopinath R. Pregabalin versus tramadol for postoperative pain management in patients undergoing lumbar laminectomy: a randomized, double-blinded, placebocontrolled study. J Pain Res 2013;6:471-8.

22. Nitta R, Goyagi T, Nishikawa T. Combination of oral clonidine and intravenous low-dose ketamine reduces the consumption of postoperative patientcontrolled analgesia morphine after spine surgery. Acta Anaesthesiol Taiwan 2013;51:14-7.
23. Hwang W, Lee J, Park J, Joo J. Dexmedetomidine versus remifentanil in postoperative pain control after spinal surgery: a randomized controlled study. BMC Anesthesiol 2015;15:21.

24. Choi YS, Shim JK, Song JW, Kim JC, Yoo YC, Kwak YL. Combination of pregabalin and dexamethasone for postoperative pain and functional outcome in patients undergoing lumbar spinal surgery: a randomized placebo-controlled trial. Clin J Pain 2013;29:914.

25. Urban MK, Labib KM, Reid SC, et al. Pregabalin did not improve pain management after spinal fusions. HSS J 2018;14:41-6.

26. Turan A, Babazade R, Kurz A, et al. Clonidine does not reduce pain or opioid consumption after noncardiac surgery. Anesth Analg 2016;123:749-57.

27. Ozgencil E, Yalcin S, Tuna H, Yorukoglu D, Kecik Y. Perioperative administration of gabapentin 1,200 $\mathrm{mg}$ day-1 and pregabalin $300 \mathrm{mg}$ day-1 for pain following lumbar laminectomy and discectomy: a randomised, double-blinded, placebo-controlled study. Singapore Med J 2011;52:883-9.

28. Grant MC, Betz M, Hulse M, et al. The effect of preoperative pregabalin on postoperative nausea and vomiting: a meta-analysis. Anesth Analg 2016;123:1100-7. 